\title{
EVIDENCE OF HOMOSUBTYPIC BUT NOT HETEROSUBTYPIC IMMUNITY IN CHILDREN AFTER WILD-TYPE INFLUENZA ILLNESS
}

\author{
C. Ambrose ${ }^{1}, \mathrm{X} . \mathrm{Wu}^{1}$, R. Pilsudski ${ }^{2}$ \\ ${ }^{I}$ MedImmune, LLC, Gaithersburg, MD, USA, ${ }^{2}$ AzstraZeneca, Paris, France
}

Background and aims: Cross-protection between different influenza A subtypes (heterosubtypic immunity) has been shown in animals after infection with wild-type influenza and administration of some types of influenza vaccines. However, data regarding protective heterosubtypic immunity in humans are limited. The aim of this analysis is to examine data from unvaccinated subjects followed in multiyear, culture-confirmed vaccine efficacy studies for evidence of heterosubtypic protection.

Methods: Four 2-year studies of children aged 6-71 months were analyzed. Placebo recipients were separated into cohorts based on whether the subject experienced culture-confirmed influenza illness in year 1. The incidence of influenza illness by subtype per cohort was calculated for year 2. Subjects with confirmed illness in year 1 were compared with subjects with no evidence of any influenza illness in year 1.

\section{Results:}

\begin{tabular}{|c|c|c|c|c|}
\hline \multicolumn{5}{|c|}{ Table. Comparisons Evaluating Heterosubtypic, Heterotypic, and Homosubtypic Immunity } \\
\hline Study (Age) & Year 1 Illness & Year 2 IIIness & Incidence in Year 2 & \\
\hline \multicolumn{4}{|c|}{ Heterosubtypic Immunity } & $P^{*}$ \\
\hline \multirow{2}{*}{$\begin{array}{l}\text { Vesikari et al } \\
(6-35 \mathrm{mo})\end{array}$} & $\mathrm{A} / \mathrm{H} 1 \mathrm{~N} 1$ & $\mathrm{~A} / \mathrm{H} 3 \mathrm{~N} 2$ & $7 / 39(17.9 \%)$ & \multirow{2}{*}{0.55} \\
\hline & No influenza illness & $\mathrm{A} / \mathrm{H} 3 \mathrm{~N} 2$ & $92 / 398(23.1 \%)$ & \\
\hline \multirow{2}{*}{$\begin{array}{l}\text { Tam et al } \\
(12-35 \mathrm{mo})\end{array}$} & $\mathrm{A} / \mathrm{H} 1 \mathrm{~N} 1$ & $\mathrm{~A} / \mathrm{H} 3 \mathrm{~N} 2$ & $3 / 40(7.5 \%)$ & \multirow{2}{*}{$>0.99$} \\
\hline & No influenza illness & $\mathrm{A} / \mathrm{H} 3 \mathrm{~N} 2$ & $40 / 410(9.8 \%)$ & \\
\hline \multirow{2}{*}{$\begin{array}{c}\text { Bracco et al } \\
(6-35 \mathrm{mo})\end{array}$} & $\mathrm{A} / \mathrm{H} 3 \mathrm{~N} 2$ & $\mathrm{~A} / \mathrm{H} 1 \mathrm{~N} 1$ & $7 / 55(12.7 \%)$ & \multirow{2}{*}{0.02} \\
\hline & No influenza illness & $\mathrm{A} / \mathrm{H} 1 \mathrm{~N} 1$ & $11 / 280(3.9 \%)$ & \\
\hline \multicolumn{4}{|c|}{ Heterotypic Immunity } & $P^{*}$ \\
\hline \multirow{2}{*}{$\begin{array}{c}\text { Vesikari et al } \\
(6-35 \mathrm{mo})\end{array}$} & $\mathrm{A} / \mathrm{H} 1 \mathrm{~N} 1$ & $\mathrm{~B}$ & $4 / 39(10.3 \%)$ & \multirow{2}{*}{0.53} \\
\hline & No influenza illness & B & $30 / 398(7.5 \%)$ & \\
\hline \multirow{2}{*}{$\begin{array}{l}\text { Tam et al } \\
(12-35 \mathrm{mo})\end{array}$} & $\mathrm{A} / \mathrm{H} 1 \mathrm{~N} 1$ & B & $2 / 40(5.0 \%)$ & \multirow{2}{*}{0.32} \\
\hline & No influenza illness & $\mathrm{B}$ & $11 / 410(2.7 \%)$ & \\
\hline \multicolumn{4}{|c|}{ Homosubtypic Immunity } & Protection $(95 \% \mathrm{Cl})$ \\
\hline \multirow{2}{*}{$\begin{array}{l}\text { Tam et } \mathrm{al}^{+} \\
(12-35 \mathrm{mo})\end{array}$} & $\mathrm{A} / \mathrm{H} 1 \mathrm{~N} 1$ & $\mathrm{~A} / \mathrm{H} 1 \mathrm{~N} 1$ & $0 / 40(0 \%)$ & \multirow{4}{*}{$\begin{array}{c}(-1453,100) \\
87 \% \\
(22,100)\end{array}$} \\
\hline & No influenza illness & $\mathrm{A} / \mathrm{H} 1 \mathrm{~N} 1$ & $4 / 410(1.0 \%)$ & \\
\hline \multirow{2}{*}{$\begin{array}{l}\text { Belshe et } \mathrm{al}^{\ddagger} \\
(15-71 \mathrm{mo})\end{array}$} & $\mathrm{A} / \mathrm{H} 3 \mathrm{~N} 2$ & $\mathrm{~A} / \mathrm{H} 3 \mathrm{~N} 2$ & $1 / 52(1.9 \%)$ & \\
\hline & No influenza illness & $\mathrm{A} / \mathrm{H} 3 \mathrm{~N} 2$ & $52 / 363(14.3 \%)$ & \\
\hline \multicolumn{4}{|c|}{$\begin{array}{l}\text { "Fisher exact test. } \\
\text { 'A/H1N1 strains in years } 1 \text { and } 2 \text { were antigenically similar. } \\
\text { "A/H3N2 strains in years } 1 \text { and } 2 \text { were antigenically distinct. }\end{array}$} & \\
\hline
\end{tabular}

Conclusions: In this analysis of the 2-year incidence of culture-confirmed influenza illness in young children, there was evidence of homosubtypic protection induced by wild-type influenza illness; however, there was no evidence of heterotypic or heterosubtypic protection. These results support previous observations that children may not develop lasting, clinically significant heterosubtypic protection after wildtype influenza infection.

References: Belshe RB, et al. J Pediatr. 2000;136(2):168-175.Bracco Neto H, et al. Pediatr Infect Dis J. 2009;28(5):365-371.Tam JS, et al. Pediatr Infect Dis J. 2007;26(7):619-628.Vesikari T, et al. Pediatrics. 2006;118(6):2298-2312.

Sponsored by MedImmune, LLC. 